\title{
Solution of two-dimensional
}

\section{time-fractional Burgers equation with high and low Reynolds numbers}

\author{
Wen Cao ${ }^{1}$, Qinwu Xu ${ }^{2 *}$ and Zhoushun Zheng ${ }^{1}$
}

\begin{tabular}{l}
\hline${ }^{*}$ Correspondence: \\
xuqinwu@nju.edu.cn \\
2Department of Mathematics, \\
Nanjing University, Nanjing, Jiangsu \\
210093, P.R. China \\
Full list of author information is \\
available at the end of the article
\end{tabular}

available at the end of the article

\begin{abstract}
Burgers' equation frequently appears in the study of turbulence theory, as well as some other scientific fields. High and low Reynolds numbers play important roles in both modeling and numerical simulation. In this paper, we apply a numerical scheme to solve a two-dimensional time-fractional Burgers equation. The key feature of the proposed method is formed by combining the discontinuous Galerkin method to spatial variables and a finite difference scheme to temporal variables. The corresponding numerical analysis is also presented. Several numerical tests are carried out to demonstrate the theoretical analysis and we present a shock wave phenomenon of the new Burgers model.
\end{abstract}

MSC: $26 \mathrm{~A} 33 ; 34 \mathrm{~A} 08$

Keywords: fractional calculus; time-fractional Burgers' equation; shock wave; numerical simulation

\section{Introduction}

The Burgers model, usually applied in the study of turbulence, is a very important nonlinear partial differential equation which has been widely investigated by researchers of both theory and computation aspects. In the classical sense, a one-dimensional Burgers equation is of the following simple form $[1,2]$ :

$$
\frac{\partial u}{\partial t}+u \frac{\partial u}{\partial x}=v \frac{\partial^{2} u}{\partial x^{2}}
$$

where $v=1 / \operatorname{Re}$ and Re indicates the value of the Reynolds number. $u(x, t)$ represents the speed of fluid media at time-space position $(x, t)$. When the diffusion term is absent (i.e., the Reynolds number becomes very large), the Burgers equation becomes the inviscid Burgers equation as follows: $\frac{\partial u}{\partial t}+u \frac{\partial u}{\partial x}=v \frac{\partial^{2} u}{\partial x^{2}} \approx 0$. This is a prototype for conservation equations that can develop discontinuities (also called shock waves) [1]. In recent years, the study of Burgers' equation has not only focused on fluid dynamics, but also included nonlinear acoustics, gas dynamics, traffic flow, and so on (see [3, 4] and references therein). With the assistance of Cole-Hopf transformations [5], more and more mathematical analysis tools and computational skills can easily be developed to find more inherent characteristics of Burgers' equation. For instance, Hoffman et al. give a distributed approximating

(c) The Author(s) 2017. This article is distributed under the terms of the Creative Commons Attribution 4.0 International License (http://creativecommons.org/licenses/by/4.0/), which permits unrestricted use, distribution, and reproduction in any medium, provided you give appropriate credit to the original author(s) and the source, provide a link to the Creative Commons license, and indicate if changes were made. 
functional method to solve Burgers' equation for relatively large Reynolds numbers in [6], where a nonlinear transform is applied to generating a nonuniform mesh so that tiny step sizes are given near the boundary to catch the satisfying approximation of shock waves. In [7], the multiple-front solutions of a class of coupled Burgers systems is studied by tanh-coth method and the Cole-Hopf transformation. In [8], the solitary solution of Burgers' equation is studied by a modified exponential-function method. This method seems to work perfectly for the integer-order Burgers model because the fractional exponential function expansion is based on the smoothness of the solution. In [9], the Crank-Nicolson method for solving Burgers' equation is discussed. The advantage of a direct full-discrete method is that it can get rid of Cole-Hopf transforms and numerical analysis can be given analogically with usual cases. Much effort has been done since the 1980s for numerical methods of the conventional Burgers equation.

Fractional calculus is not a new subject. It first appeared in the discussion by mathematicians Leibniz and l'Hospital. However, its theoretical growth took up several hundreds years before people found that it might have very important potential applications [10]. In many scientific and engineering fields, such as theoretical physical problems [11], diffusion processes [12], vibration and control [13], oscillating dynamical systems [14], thermal conductivity [15], rheological models [16], quantum models [17], etc., fractional derivative models manifest much better and describe real-world data more accurate than integer-order models do. Fractional derivative models not only preserve the basic features of considered problems, but also give more parameters to predict other inner properties of the model, especially for the problems involving spatial dependence and temporal memory [18]. For instance, based on the previous research on diffusion models, it is found that the temporal fractional derivative with order one is considered to be a critical value. Then those models with orders of temporal fractional derivative of less than one directly lead to subdiffusion processes, while the models containing orders greater than one indicate the superdiffusion processes. Therefore, fractional derivative models provide an effective way to model anomalous diffusion problems.

Nowadays, fractional calculus modeling methods are considered as powerful techniques to handle physical and engineering problems with memory feature and nonlocal property, due to the natural structure of fractional integrals and derivatives making them a global and weak singular integration [10]. Nevertheless, the study of Burgers' equation is fruitful and the literature on these models with fractional derivatives is still scarce. Especially for the multiple-dimensional fractional Burgers model, there are many concrete problems that need to be solved. Therefore, it shall be meaningful to further examine Burgers models with various fractional derivatives. Since the 1990s, numerical methods for fractional differential equations have undergone considerable development, due to the reason that more and more classic models are reconsidered by using a fractional calculus framework. Thus, in this paper, we shall consider a class of two-dimensional time-fractional Burgers equations over bounded domain with high and low Reynolds numbers, and further study the dynamic behaviors through numerical simulations.

Numerical methods applied to a fractional Burgers equation include the finite difference method, the integral transform method, the variational method, and some others. As mentioned, some of them become easier under a Cole-Hopf transform; i.e., see [19]. For instance, in [20] a fractional Burgers equation with half-order damping term is considered. It is verified that if the coefficient of the half-order derivative is also small, as is 
usually the case, the evolution comprises three stages, namely a lossless near field, an intermediate Burgers region, and a hereditary far field. In [21], the series expansion method is applied to a local fractional derivative to obtain the solutions of both homogeneous and nonhomogeneous transport equations. The finite difference and spectral methods are applied to numerical simulations. In [22], the Adomian decomposition method is applied to solve the fractional KdV-Burgers equation. The decomposition methods are powerful to provide a series expansion which eventually converges to the true solution. In [23], the Adomian decomposition method is applied to study a class of coupled Burgers equations with Caputo derivatives equipped on both temporal and spatial variables. The obtained numerical solutions are of a form of rapidly convergent series and easily computable components. In [24], a nonlinear fractional Burgers problem is approximated by finite volume schemes of order one in space and, also, in time. It is proved that the finite volume solution converges to a weak entropic solution as the step size vanishes finally. In $[25,26]$ new spectral methods are proposed for fractional differential equations and high efficiency property of the methods is demonstrated through numerical examples. More numerical studies on time- and/or space-fractional Burgers equations can be found in [27-33]. Recently, one interesting branch of numerical study of fractional Burgers equations has emerged: the implementation of the discontinuous Galerkin method (DGM) [34-40]. The DGM has several advantages to reach this goal: (1) it behaves well if the solution of Burgers' equation is regular enough under certain initial and parameter settings; (2) when a shock wave solution appears, the DGM can handle high-effective numerical approximations near discontinuities [41]. Unfortunately, the results for the DGM in fractional Burgers equations, as well as many nonlinear fractional integrable systems, are not fruitful. This became the major motivation to carry out the current work.

The rest of this paper is organized as follows. In Section 2, we briefly introduce fractional integrals and derivatives to make this article self-contained. In Section 3, we propose a two-dimensional time-fractional Burgers equation and present a hybrid numerical scheme based on the DGM and the finite difference scheme. In Section 4, two numerical examples are given and numerical simulations are carried out and discussed. Finally, conclusions are drawn and some future plans are suggested in Section 5.

\section{Preliminaries}

We briefly recall some definitions and properties of fractional integrals and derivatives. More details of them can be found in popular monographs [10]. Let $\Gamma(\cdot)$ be the gamma function and let $\alpha>0$ be a real number. By extending the usual multi-fold integration of integer-order, it is natural to have the following fractional integral operator.

Definition 1 (see [10]) The left Riemann-Liouville fractional integral of order $\alpha>0$ of a function $u(t), 0 \leq t \leq+\infty$ is defined as

$$
\left(I_{0+}^{\alpha} u\right)(t)=\frac{1}{\Gamma(\alpha)} \int_{0}^{t}(t-s)^{\alpha-1} u(s) d s,
$$

provided the integral is finite.

Borrowing the fact that differentiation and integration operations are mutually inverse in general, there are two basic ways to define fractional derivatives, which are, respectively, called the Riemann-Liouville sense and the Caputo sense. 
Definition 2 (see [10]) The left Riemann-Liouville fractional derivative of order $n-1<$ $\alpha<n$ of a function $u(t)$ is defined as

$$
\left(D_{0+}^{\alpha} u\right)(t)=\frac{1}{\Gamma(n-\alpha)}\left(\frac{d^{n}}{d t^{n}}\right) \int_{0}^{t}(t-s)^{n-\alpha-1} u(s) d s,
$$

provided the right side of the identity is finite.

Definition 3 (see [10]) The left Caputo fractional derivative of order $n-1<\alpha<n$ of a function $u(t)$ is defined as

$$
\left({ }^{c} D_{0+}^{\alpha} u\right)(t)=\frac{1}{\Gamma(n-\alpha)} \int_{0}^{t}(t-s)^{n-\alpha-1} u^{(n)}(s) d s
$$

provided the right side of the identity is finite.

In most of the literature, it is verified that models with Riemann-Liouville derivative need some nonlocal initial condition to guarantee the existence and regularity of solution. However, models with Caputo derivative can inherit the conventional initial and boundary conditions of corresponding integer-order models. Therefore, we give the partial fractional derivative of a function $u(x, y, t)$ in the Caputo sense below.

Definition 4 (see [10]) The left partial fractional derivative of order $n-1<\alpha<n$ of a function $u(x, y, t)$, with respective to variable $t$, in the Caputo sense, is defined as

$$
\partial_{t}^{\alpha} u(x, y, t)=\frac{1}{\Gamma(n-\alpha)} \int_{0}^{t}(t-s)^{n-\alpha-1} \frac{\partial^{n} u(x, y, s)}{\partial s^{n}} d s
$$

provided the right side of the identity is finite.

\section{Model and numerical method}

Let $\Omega=[-L, L] \times[-L, L]$ be a bounded domain from $\mathbb{R}^{2}, L>0$. $\partial \Omega$ denotes its boundary. Let $0<t<T$. Then $\mathcal{D}=\Omega \times(0, T)$ and $\mathcal{S}=\partial \Omega \times(0, T)$ are the parabolic boundaries, where $0<T<\infty$. In what follows, we shall consider a time-fractional Burgers equation

$$
\partial_{t}^{\alpha} u+u\left(u_{x}+u_{y}\right)=v \Delta u, \quad 0<\alpha<1,
$$

where $\Delta=\frac{\partial^{2}}{\partial x^{2}}+\frac{\partial^{2}}{\partial y^{2}}, v>0$. We consider the cases with Reynolds number. Let $\operatorname{Re} \in$ $[1,1,000]$. To make (6) solvable, we impose the initial and boundary conditions

$$
u(x, y, 0)=\varphi(x, y), \quad(x, y) \in \Omega ; \quad u(x, y, t)=0, \quad(x, y, t) \in \mathcal{S} .
$$

\subsection{Conservation of solution}

Now we start our discussion by recalling the fact that solving (6) with a closed form solution is impossible, thus we shall adopt some useful way to find the evidence that a solution with certain regularity exists. The fundamental skill is borrowed from the projection method. Let $\phi(x, y) \in \mathcal{C}_{0}^{\infty}(\Omega)$, where $\mathcal{C}_{0}^{\infty}(\Omega)$ denotes the set of all sufficiently smooth functions with compact support defined in $\Omega$. 
In the case of high Reynolds number, i.e., $\operatorname{Re} \gg 1$, we have $\frac{1}{\operatorname{Re}} \Delta u \approx 0$. This motivates us to only study

$$
\partial_{t}^{\alpha} u+u\left(u_{x}+u_{y}\right)=0
$$

We similarly call it an inviscid time-fractional Burgers equation. Let us integrate (8) over domain $\Omega$. Then

$$
\iint_{\Omega} \partial_{t}^{\alpha} u d x d y+\iint_{\Omega} u\left(u_{x}+u_{y}\right) d x d y=0 .
$$

Considering the second part of equation (9) and using the definition of divergence and Gauss's theorem, we have

$$
\iint_{\Omega} u\left(u_{x}+u_{y}\right) d x d y=\iint_{\Omega} \nabla \cdot \mathcal{G} d x d y=\int_{\partial \Omega} \mathcal{G} \cdot \mathbf{n} d s,
$$

where $\mathcal{G}=\left(\frac{u^{2}}{2}, \frac{u^{2}}{2}\right)$ is the flux through the boundary of the domain, and $\mathbf{n}$ is the unit outward normal vector. Using the boundary condition (7), we have $\left.\frac{u^{2}}{2}\right|_{\partial \Omega}=0$. We deduce

$$
\partial_{t}^{\alpha} \iint_{\Omega} u d x d y=\iint_{\Omega} \partial_{t}^{\alpha} u d x d y=-\iint_{\Omega} u\left(u_{x}+u_{y}\right) d x d y=0 .
$$

By the definition of the Caputo derivative, we get

$$
\frac{\partial}{\partial t} \iint_{\Omega} u d x d y=0
$$

Next, we consider a similar feature for viscous Burgers equations. Let $\mathcal{H}=\left(u_{x}, u_{y}\right)$. Integrating both sides of the equation over $\Omega$, the viscous part then becomes

$$
\iint_{\Omega} v \Delta u d x d y=v \iint_{\Omega} \nabla \cdot \mathcal{H} d x d y=v \int_{\partial \Omega} \mathcal{H} \cdot \mathbf{n} d s
$$

Assuming $u$ is smooth near the boundary and, $\forall(x, y) \in \Omega \backslash \partial \Omega, \phi(x, y)>0$, obviously $\mathcal{H}$. $\mathbf{n}<0$. Then we have

$$
\partial_{t}^{\alpha} \iint_{\Omega} u d x d y=v \int_{\partial \Omega} \mathcal{H} \cdot \mathbf{n} d s<0
$$

By the definition of Caputo derivative, we get

$$
\frac{\partial}{\partial t} \iint_{\Omega} u d x d y<0
$$

Otherwise, if, $\forall(x, y) \in \Omega \backslash \partial \Omega, \phi(x, y)<0$, we have

$$
\frac{\partial}{\partial t} \iint_{\Omega} u d x d y>0
$$

Equations (15)-(16) show the dissipative property of the viscous term. It is also called dissipative term, which makes the solution approach 0 . The dissipation strength is determined by the Reynolds number $\operatorname{Re}=\frac{1}{v}$. For $\operatorname{Re} \gg 1$, the equation approximates a pure 
wave equation and the energy is almost conserved. While $\operatorname{Re} \sim 1$, the dissipative term will be the leading part. The velocity of flow shall decay due to the impact of the viscous force. The flow field becomes stable eventually.

By the property of Caputo derivative, the time-fractional Burgers equation keeps the conservation or dissipation property of the classical Burgers equation.

\subsection{Discontinuous Galerkin method}

In this part, we propose a numerical scheme based on the full discretization which is of finite differences for temporal variables and the DGM for spatial variables. The basic advantage of the DGM is that the shock wave solution of Burgers' equation can be better approximated.

\subsubsection{Semi-discretization scheme}

We assume that the domain $\Omega$ is well approximated by the computational domain $\Omega_{h}$. Let $\mathcal{T}_{h}$ be a quasi-uniform family of triangulations domain $\Omega_{h}$ parameterized by $h>0$. For any triangle $D^{k} \in \mathcal{T}_{h}$, we define $h_{k}:=\operatorname{diam}\left(D^{k}\right),(k=1,2, \ldots, K), h=\max _{D^{k} \in \mathcal{T}_{h}} h_{k}$. Then

$$
\Omega \simeq \Omega_{h}=\bigcup_{k=1}^{K} D^{k} .
$$

We define

$$
\begin{aligned}
& \mathcal{E}_{h}^{I}:=\text { set of all interior edges of } \mathcal{T}_{h} ; \\
& \mathcal{E}_{h}^{B}:=\text { set of all boundary edges of } \mathcal{T}_{h} \text { on } \partial \Omega .
\end{aligned}
$$

For the construction of the discontinuous Galerkin scheme, we introduce the following broken Sobolev space for given real number $s$ :

$$
H^{s}\left(\Omega_{h}\right)=\left\{v \in L^{2}(\Omega): \forall k=1,2, \ldots, K,\left.v\right|_{D^{k}} \in H^{s}\left(D^{k}\right)\right\}
$$

equipped with the broken Sobolev norm

$$
\|v\|_{H\left(\Omega_{h}\right)}=\left(\sum_{k=1}^{K}\|v\|_{H^{s}\left(D^{k}\right)}^{2}\right)^{1 / 2} .
$$

In order to establish the local discontinuous Galerkin discretization, we introduce two auxiliary variables $p=\frac{\partial u}{\partial x}, q=\frac{\partial u}{\partial y}$. Then we have

$$
\begin{aligned}
& \partial_{t}^{\alpha} u+u\left(u_{x}+u_{y}\right)=\sqrt{v}\left(p_{x}+q_{y}\right), \\
& p=\sqrt{v} u_{x}, \\
& q=\sqrt{v} u_{y} .
\end{aligned}
$$


Given a triangulation $\mathcal{T}_{h}$, we try to find $u_{h}, p_{h}, q_{h} \in H^{s}\left(\Omega_{h}\right)$, such that, for any element $D^{k}$ and $\phi_{i}^{k}, \psi_{i}^{k}, \lambda_{i}^{k} \in H^{s}\left(D^{k}\right)$, the following equations hold:

$$
\begin{aligned}
& \iint_{D^{k}} \partial_{t}^{\alpha} u_{h} \phi_{i}^{k}(x, y) d x d y \\
& +\iint_{D^{k}}\left(\frac{1}{2}\left(\frac{\partial u^{2}}{\partial x}+\frac{\partial u^{2}}{\partial y}\right)-\sqrt{v} \nabla \cdot\left(p_{h}, q_{h}\right)\right) \phi_{i}^{k}(x, y) d x d y=0, \\
& \iint_{D^{k}} p_{h} \psi_{i}^{k}(x, y) d x d y=\iint_{D^{k}} \sqrt{v} \frac{\partial u_{h}}{\partial x} \psi_{i}^{k}(x, y) d x d y, \\
& \iint_{D^{k}} q_{h} \lambda_{i}^{k}(x, y) d x d y=\iint_{D^{k}} \sqrt{v} \frac{\partial u_{h}}{\partial y} \lambda_{i}^{k}(x, y) d x d y .
\end{aligned}
$$

To avoid double values at edges, we define the numerical flux for values at edges $u_{h}^{*}=$ $f_{1}\left(u_{h}^{-}, h_{h}^{+}\right), \mathcal{F}_{h}^{*}=f_{2}\left(\mathcal{F}^{-}, \mathcal{F}^{+}\right)$, where $\mathcal{F}_{h}=\left(\frac{u_{h}^{2}}{2}-\sqrt{v} p_{h}, \frac{u_{h}^{2}}{2}-\sqrt{v} q_{h}\right)^{\top}$ and we define $\hat{n}=\left(n_{x}, n_{y}\right)$. By integration by part, we obtain

$$
\begin{aligned}
& \iint_{D^{k}} \partial_{t}^{\alpha} u_{h} \phi_{i}^{k}(x, y) d x d y=-\int_{\partial D^{k}} \hat{n} \cdot \mathcal{F}_{h}^{*} \phi_{i}^{k}(x, y) d s+\iint_{D^{k}} \mathcal{F}_{h} \cdot \nabla \phi_{i}^{k}(x, y) d x d y, \\
& \iint_{D^{k}} p_{h} \psi_{i}^{k}(x, y) d x d y=\sqrt{v} \int_{\partial D^{k}} \hat{n}_{x} u_{h}^{*} \psi_{i}^{k}(x, y) d s-\sqrt{v} \iint_{D^{k}} u_{h}\left(\psi_{i}^{k}(x, y)\right)_{x} d x d y, \\
& \iint_{D^{k}} q_{h} \lambda_{i}^{k}(x, y) d x d y=\sqrt{v} \int_{\partial D^{k}} \hat{n}_{y} u_{h}^{*} \lambda_{i}^{k}(x, y) d s-\sqrt{v} \iint_{D^{k}} u_{h}\left(\lambda_{i}^{k}(x, y)\right)_{y} d x d y .
\end{aligned}
$$

To define the numerical flux function $f_{1}, f_{2}$, let $\{\{a\}\}$ denote average of $a$, that is, $\{\{a\}\}=$ $\frac{a^{-}+a^{+}}{2}$. Let $[[a]]$ denote the jump value of $a$, that is, $[[a]]=a^{+}-a^{-}$. For the flux associated with the diffusion term, we define $u_{h}^{*}=\left\{\left\{u_{h}\right\}\right\}$. For the convection term, we introduce the local monotone Lax-Friedrichs flux defined by [17]. Then

$$
\mathcal{F}_{h}^{*}=\left\{\left\{\mathcal{F}_{h}\right\}\right\}-\frac{C}{2}[[u]]
$$

where $C=\max _{s \in\left[u_{h}^{-}, u_{h}^{+}\right]}\left|\frac{\partial \mathcal{F}_{h}}{\partial u_{h}}\right|$. The local monotone Lax-Friedrichs numerical flux is a particularly convenient choice of numerical flux because it can easily be applied to any nonlinear hyperbolic system, it is simple to compute, and it yields good results, although there are many other numerical fluxes which could also be used [24].

We consider discretization of the fractional Burgers equation in a piecewise polynomial space of degree $N$. Assuming $\mathcal{L}_{j}^{k}(x, y)$ s are Lagrange polynomials defined on $D^{k}$, $j=1,2, \ldots, N_{p}, N_{p}=\frac{(N+1)(N+2)}{2}$, they form a group of bases for polynomial space of degree $N$ in $D^{k}$. Then, for any element $D^{k}(k=1,2, \ldots, K)$, we have

$$
\left.u_{h}\right|_{D_{k}}=\sum_{j=1}^{N_{p}} u_{j}^{k} \mathcal{L}_{j}^{k}(x, y) .
$$

Replacing the test functions $\phi_{i}^{k}, \psi_{i}^{k}, \lambda_{i}^{k}$ in the above equations with polynomial test functions $\mathcal{L}_{i}^{k}(x, y)$, the semi-discretized scheme is derived. We have

$$
\mathbf{M}^{\mathbf{k}} \partial_{t}^{\alpha} U_{h}^{k}=-\mathbf{B}^{k} \mathcal{F}_{h}^{k *}+\mathbf{S}^{k} \mathcal{F}_{h}^{k}
$$




$$
\begin{aligned}
& \mathbf{M}^{\mathbf{k}} P_{h}^{k}=\sqrt{\nu} \mathbf{B}_{x}^{k} U_{h}^{k *}+\sqrt{\nu} \mathbf{S}_{x}^{k} U_{h}^{k}, \\
& \mathbf{M}^{\mathbf{k}} Q_{h}^{k}=\sqrt{\nu} \mathbf{B}_{y}^{k} U_{h}^{k *}+\sqrt{\nu} \mathbf{S}_{y}^{k} U_{h}^{k},
\end{aligned}
$$

where $U_{h}^{k}$ is a column vector with $N_{p}$ elements, $U_{h, j}^{k}=u_{h, j}^{k}$, and $\mathcal{F}_{h}^{k *}$ and $U_{h}^{k *}$ are the corresponding numerical fluxes defined at the boundary of element $k$.

$$
\begin{aligned}
& \mathbf{M}_{i j}^{k}=\iint_{D^{k}} \mathcal{L}_{j}^{k} \mathcal{L}_{i}^{k} d x d y, \quad \mathbf{B}_{i j}^{k}=\int_{\partial D^{k}} \mathcal{L}_{i}^{k} \mathcal{L}_{i}^{k} \hat{n} d s, \quad \mathbf{S}_{i j}^{k}=\iint_{D^{k}} \mathcal{L}_{j}^{k} \nabla \mathcal{L}_{i}^{k} d x d y, \\
& \left(\mathbf{B}_{x}^{k}\right)_{i j}=\int_{\partial D^{k}} \hat{n}_{x} \mathcal{L}_{j}^{k} \mathcal{L}_{i}^{k} d s, \quad\left(\mathbf{B}_{y}^{k}\right)_{i j}=\int_{D^{k}} \hat{n}_{y} \mathcal{L}_{i}^{k} \mathcal{L}_{i}^{k} d s, \\
& \left(\mathbf{S}_{x}^{k}\right)_{i j}=\iint_{D^{k}} \mathcal{L}_{j}^{k}\left(\mathcal{L}_{i}^{k}\right)_{x} d x d y, \quad\left(\mathbf{S}_{y}^{k}\right)_{i j}=\iint_{D^{k}} \mathcal{L}_{j}^{k}\left(\mathcal{L}_{i}^{k}\right)_{y} d x d y .
\end{aligned}
$$

\subsubsection{Time integration}

Compared with the classical Burgers equation, a proper discretization scheme for timefractional derivatives is important. In references, several different approximation schemes are proposed, such as the Grünwald-Letnikov scheme, $L_{1}$ approximation. The local DGM is often combined with the explicit time integration scheme, s.t. the Euler method and the Runge-Kutta method, to take its full advantages. Especially for nonlinear problems, the implicit time integration scheme will bring about great computational cost due to large degrees of freedom of the local DGM and the need to solve nonlinear equations.

For the discretization on fractional derivatives, we employ the algorithm from [42], which reads

$$
\left({ }^{c} D_{0+}^{\alpha} f\right)(t) \approx \frac{1}{(\Delta t)^{\alpha}} \sum_{\eta=0}^{N} \omega_{\eta}^{\alpha}\left(f\left(t_{N-\eta}\right)-\sum_{k=0}^{m-1} \frac{f^{(k)}(0) t^{k}}{k !}\right),
$$

where $\omega_{\eta}^{\alpha}=(-1)^{\eta}\left(\begin{array}{l}\eta \\ \alpha\end{array}\right)$, which can be obtained recursively through the following relation:

$$
\omega_{0}^{\alpha}=1, \quad \omega_{\eta}^{\alpha}=\left(1-\frac{\alpha+1}{\eta}\right) \omega_{\eta-1}^{\alpha} .
$$

We introduce the following lemma.

Lemma 1 (see [42]) Let $f \in \mathcal{C}^{1}[0, T], 0<\alpha \leq 1.0=t_{0}<t_{1}<\cdots<t_{N}=t$ with $t_{i+1}-t_{i}=\Delta t$ is a discretization of $[0, t]$. Then

$$
\left({ }^{c} D_{0+}^{\alpha} f\right)=\sum_{\nu=0}^{N} \omega_{\nu}^{\alpha} \frac{f\left(t_{N-v}\right)-f(0)}{(\Delta t)^{\alpha}}+O(\Delta t) .
$$

Finally, we obtain the numerical scheme for solving the proposed model (6). Denote the values of $U_{h}^{k}$ and $\mathcal{F}_{h}^{k}$ at time level $t_{n}$ as $\left(U_{h}^{k}\right)^{n}$ and $\left(\mathcal{F}_{h}^{k}\right)^{n}$. Combine the time discretization with the local discontinuous Galerkin scheme and equations (23)-(25) can be written

$$
\begin{aligned}
& \left(U_{h}^{k}\right)^{n+1}=(\Delta t)^{\alpha}\left(\mathbf{M}^{\mathbf{k}}\right)^{-1}\left[-\mathbf{B}^{k}\left(\mathcal{F}_{h}^{k *}\right)^{n}+\mathbf{S}^{k}\left(\mathcal{F}_{h}^{k}\right)^{n}\right]-\sum_{\eta=1}^{n} \omega_{\eta}^{\alpha}\left(U_{h}^{k}\right)^{n}, \\
& \left(P_{h}^{k}\right)^{n}=\left(\mathbf{M}^{\mathbf{k}}\right)^{-1}\left[\sqrt{\nu} \mathbf{B}_{x}^{k}\left(U_{h}^{k *}\right)^{n}+\sqrt{\nu} \mathbf{S}_{x}^{k}\left(U_{h}^{k}\right)^{n}\right],
\end{aligned}
$$


Table 1 Error and order of convergence of time discretization for example 1 ( $h=0.3$, polynomial degree $N=4$ )

\begin{tabular}{|c|c|c|c|c|c|c|}
\hline \multirow[t]{2}{*}{$\Delta t$} & \multicolumn{2}{|l|}{$\alpha=0.7$} & \multicolumn{2}{|l|}{$\alpha=0.8$} & \multicolumn{2}{|l|}{$\alpha=0.9$} \\
\hline & $\left\|e_{u}\right\|_{2}$ & Order & $\left\|e_{u}\right\|_{2}$ & Order & $\left\|e_{u}\right\|_{2}$ & Order \\
\hline $1.0 \mathrm{e}-4$ & $1.46 \mathrm{e}-04$ & - & $1.46 \mathrm{e}-04$ & - & $1.48 \mathrm{e}-04$ & - \\
\hline $5.0 e-5$ & $7.83 e-05$ & 0.90 & $7.76 \mathrm{e}-05$ & 0.91 & $7.79 e-05$ & 0.92 \\
\hline $2.5 e-5$ & $4.28 \mathrm{e}-05$ & 0.87 & $4.23 e-05$ & 0.88 & $3.97 e-05$ & 0.97 \\
\hline
\end{tabular}

Table 2 Error and order of convergence of spatial discretization for example 1

\begin{tabular}{|c|c|c|c|c|c|c|}
\hline \multirow[t]{2}{*}{$\boldsymbol{h}$} & \multicolumn{2}{|l|}{$\alpha=0.7$} & \multicolumn{2}{|l|}{$\alpha=0.8$} & \multicolumn{2}{|l|}{$\alpha=0.9$} \\
\hline & $\left\|e_{u}\right\|_{2}$ & Order & $\left\|e_{u}\right\|_{2}$ & Order & $\left\|e_{u}\right\|_{2}$ & Order \\
\hline & $N=1$ & & & & & \\
\hline 0.4 & $4.43 e-02$ & & $4.29 e-02$ & & $4.1 \mathrm{e}-2$ & - \\
\hline 0.2 & $9.89 e-03$ & 2.16 & $9.80 \mathrm{e}-03$ & 2.13 & $9.8 \mathrm{e}-3$ & 2.06 \\
\hline \multirow[t]{2}{*}{0.1} & $2.37 e-03$ & 2.06 & $2.35 \mathrm{e}-03$ & 2.06 & $2.3 e-3$ & 2.09 \\
\hline & $N=2$ & & & & & \\
\hline 0.8 & $7.78 \mathrm{e}-02$ & & $7.08 \mathrm{e}-02$ & & $6.45 e-02$ & \\
\hline 0.4 & $1.19 e-02$ & 2.71 & $1.13 e-02$ & 2.65 & $1.07 e-02$ & 2.59 \\
\hline 0.2 & $1.39 e-03$ & 3.10 & $1.40 \mathrm{e}-03$ & 3.01 & $1.30 e-03$ & 3.04 \\
\hline
\end{tabular}

$$
\left(Q_{h}^{k}\right)^{n}=\left(\mathbf{M}^{\mathbf{k}}\right)^{-1}\left[\sqrt{\nu} \mathbf{B}_{y}^{k}\left(U_{h}^{k *}\right)^{n}+\sqrt{\nu} \mathbf{S}_{y}^{k}\left(U_{h}^{k}\right)^{n}\right]
$$

\section{Numerical simulation and discussion}

Example 1 To test the performance and accuracy of the method, we consider the example with source term and exact solution

$$
\begin{cases}\partial_{t}^{\alpha} u+u\left(u_{x}+u_{y}\right)=\frac{1}{10} \Delta u+f_{\alpha}(x, y, t), & (x, y) \in \Omega, t \in(0,1] \\ u(x, y, 0)=0, & (x, y) \in \Omega, \\ \left.u(x, y, t)\right|_{\partial \Omega}=0, & t \in(0,1],\end{cases}
$$

where $\Omega=[-1,1] \times[-1,1], t \in[0,1]$. We assume the exact solution $u(x, y, t)=t^{3}\left(1-x^{2}\right)^{2}(1-$ $\left.y^{2}\right)^{2}$, so the source term is obtained. We have

$$
\begin{aligned}
f_{\alpha}(x, y, t)= & \frac{6 t^{3-\alpha}\left(1-x^{2}\right)^{2}}{\Gamma(4-\alpha)}\left(1-y^{2}\right)^{2}+4 t^{6}\left(1-x^{2}\right)^{3}\left(1-y^{2}\right)^{3}\left(x^{2} y+x y^{2}-x-y\right) \\
& -0.4 t^{3}\left[\left(y^{2}-1\right)^{2}\left(3 x^{2}-1\right)+\left(x^{2}-1\right)^{2}\left(3 y^{2}-1\right)\right] .
\end{aligned}
$$

From Table 1, we observe that, when high accuracy spatial discretization is used, the accuracy is controlled by time discretization and the finite difference scheme is of firstorder accuracy, just as indicated by Lemma 1.

To test the effectiveness and accuracy of the spatial discretization, we set the time step length to be very small and compare the errors for different spatial discretization. From Table 2, we see that the convergence order approximates 2 for $N=1$ and is close to 3 for $N=2$, which indicates that spatial discretization converges with order $N+1$. 


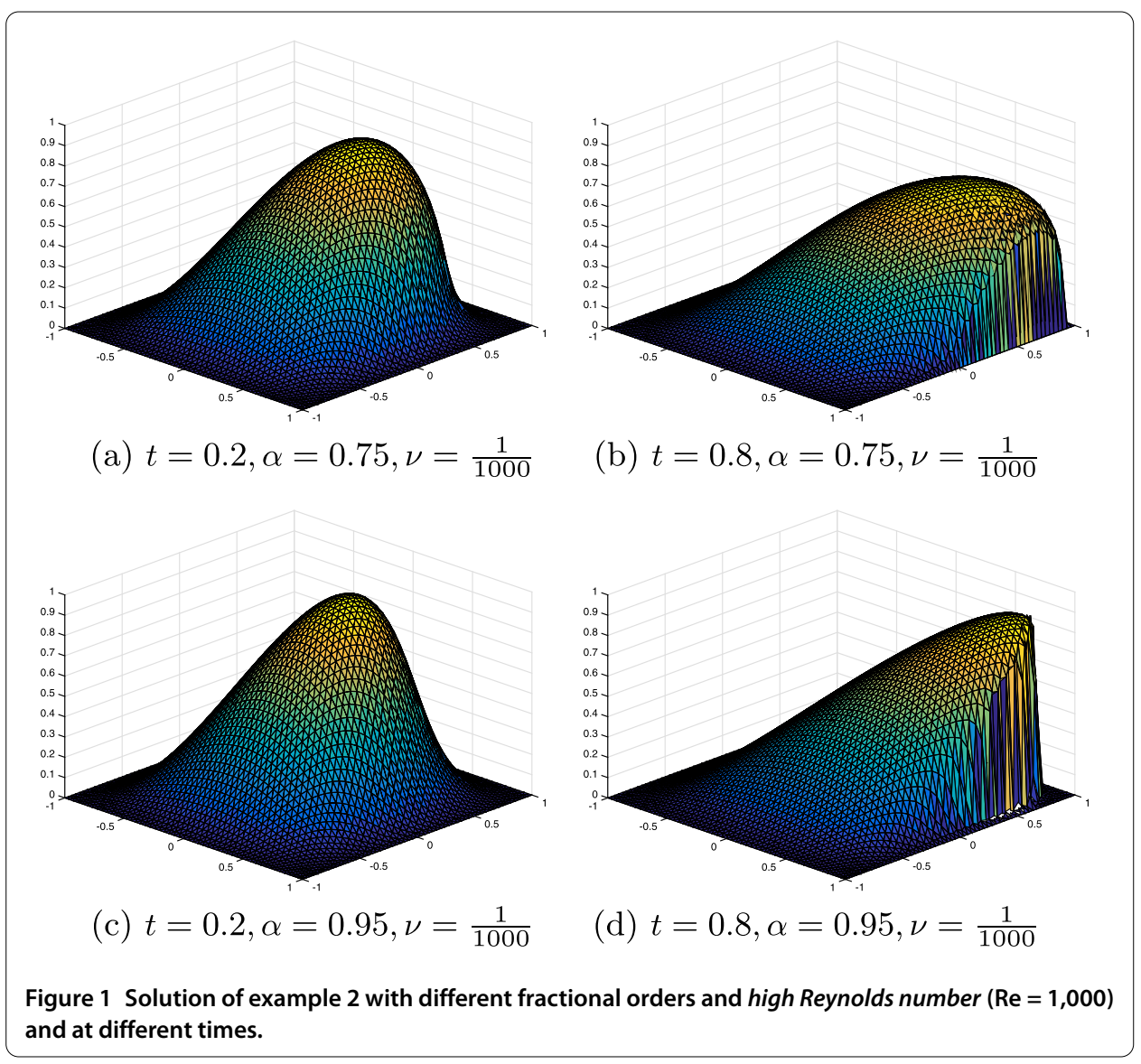

Example 2 In this example, we consider the following time-fractional Burgers equation:

$$
\begin{cases}\partial_{t}^{\alpha} u+u\left(u_{x}+u_{y}\right)=v \Delta u, & (x, y) \in \Omega, t \in(0,1] \\ u(x, y, 0)=\left(x^{2}-1\right)^{2}\left(y^{2}-1\right)^{2}, & (x, y) \in \Omega \\ \left.u\right|_{\partial \Omega}=0, & t \in(0,1]\end{cases}
$$

where all the symbols have the same meaning as in the last example.

We consider the problem in the domain $\Omega=[-1,1] \times[-1,1]$. To study the influence of the order of time-fractional derivative and the Reynolds number $\operatorname{Re}(\operatorname{Re}=1 / v)$, we solve Example 2 with different settings of $\alpha$, $v$ and illustrate phase plots at some particular moments, to show the dynamics of equation (31). The results are shown in Figures 1-3.

From Figures 1-3, the following observations can be obtained:

(1) We select three different values of $v$, which, respectively, correspond to high, moderate, and low Reynolds numbers. One can easily see that model (6) reveals a convectiondominant phenomenon with a high Reynolds number, while it shows dispersion-dominant characteristics with a low Reynolds number. This is expected by comparing with the classic Burgers equation. The fractional Burgers equation indeed preserves the fundamental property of integer-order Burgers equations.

(2) When high Reynolds numbers are considered, we solve model (6) with different orders of fractional derivatives and illustrate numerical solutions at moments $t=0.2$ and 


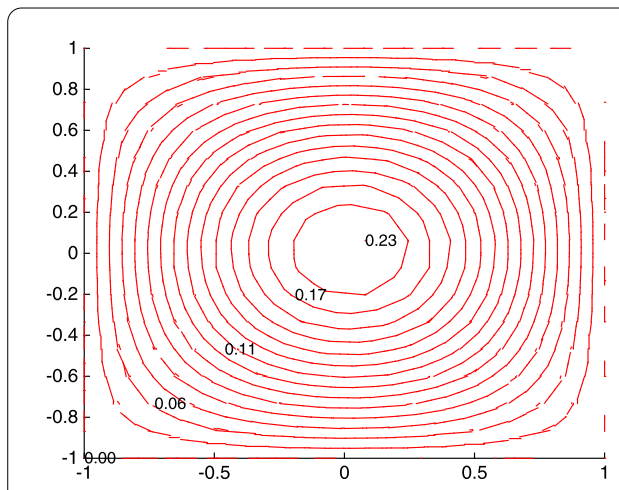

(a) $t=0.2, \alpha=0.75, \nu=1$

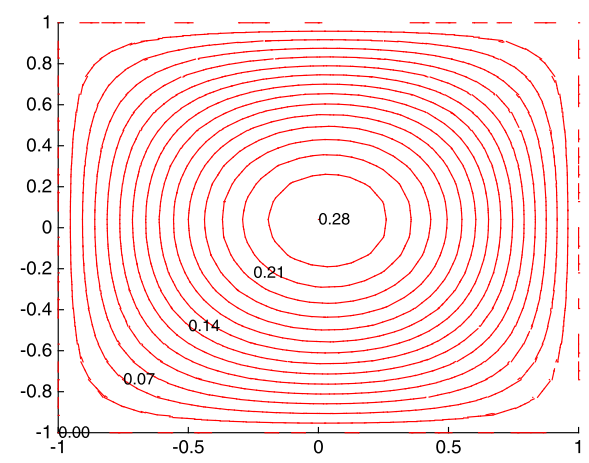

(c) $t=0.2, \alpha=0.95, \nu=1$

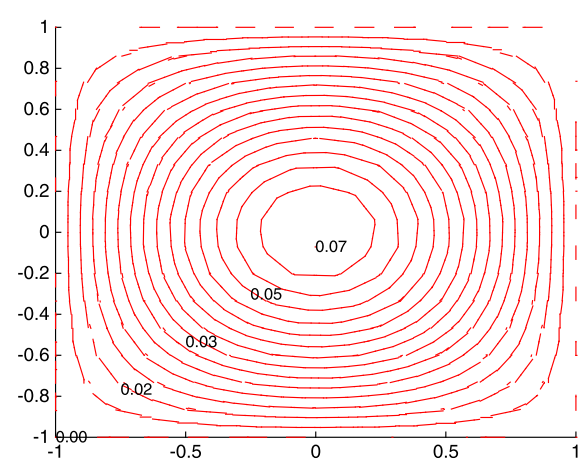

(b) $t=0.8, \alpha=0.75, \nu=1$

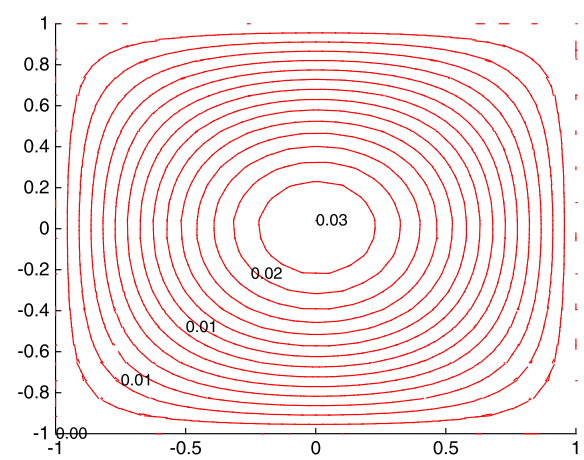

(d) $t=0.8, \alpha=0.95, \nu=1$

Figure 2 Solution of example 2 with different fractional orders and low Reynolds number $(\operatorname{Re}=1)$ and at different times.

$t=0.8$. As we can observe from Figure 1, the peak of the solution moves to the boundary rapidly and crushes at the wavefront, which is called the shock wave phenomenon in the nonlinear partial differential equation theory. Therefore, the fractional Burgers equation also can display a shock wave solution which is not globally continuously differentiable as that of the classic Burgers equation, under certain selections of parameters in the equation.

(3) When low Reynolds numbers are considered, we also solve model (6) with different orders of fractional derivatives and illustrate numerical solutions at the same moments $t=$ 0.2 and $t=0.8$. Similarly, we observe from Figure 2 that the peak of the solution moves to the boundary slowly, and it is hard to see discontinuity in the solution domain. Therefore, the fractional Burgers equation has a globally-defined solution as that of the classic inviscid Burgers equation.

(4) When a moderate Reynolds number is considered, we again solve model (6) with different orders of fractional derivatives and illustrate numerical solutions at the same moments $t=0.2$ and $t=0.8$. As we observe from Figure 3 , the peak of the solution moves to the boundary. However, due to the fact that the Reynolds number is not high enough, the numerical solution is still regular on the boundary. Thus, we clearly see that the size of the Reynolds number determines the behavior of the fractional Burgers equation.

(5) Now we make a systematic comparison on the above three plots. No matter how high or low the Reynolds number is, when the Reynolds number is fixed, model (6) diffuses relatively fast under small $\alpha$ and correspondingly slow under large $\alpha$. This agrees with the 


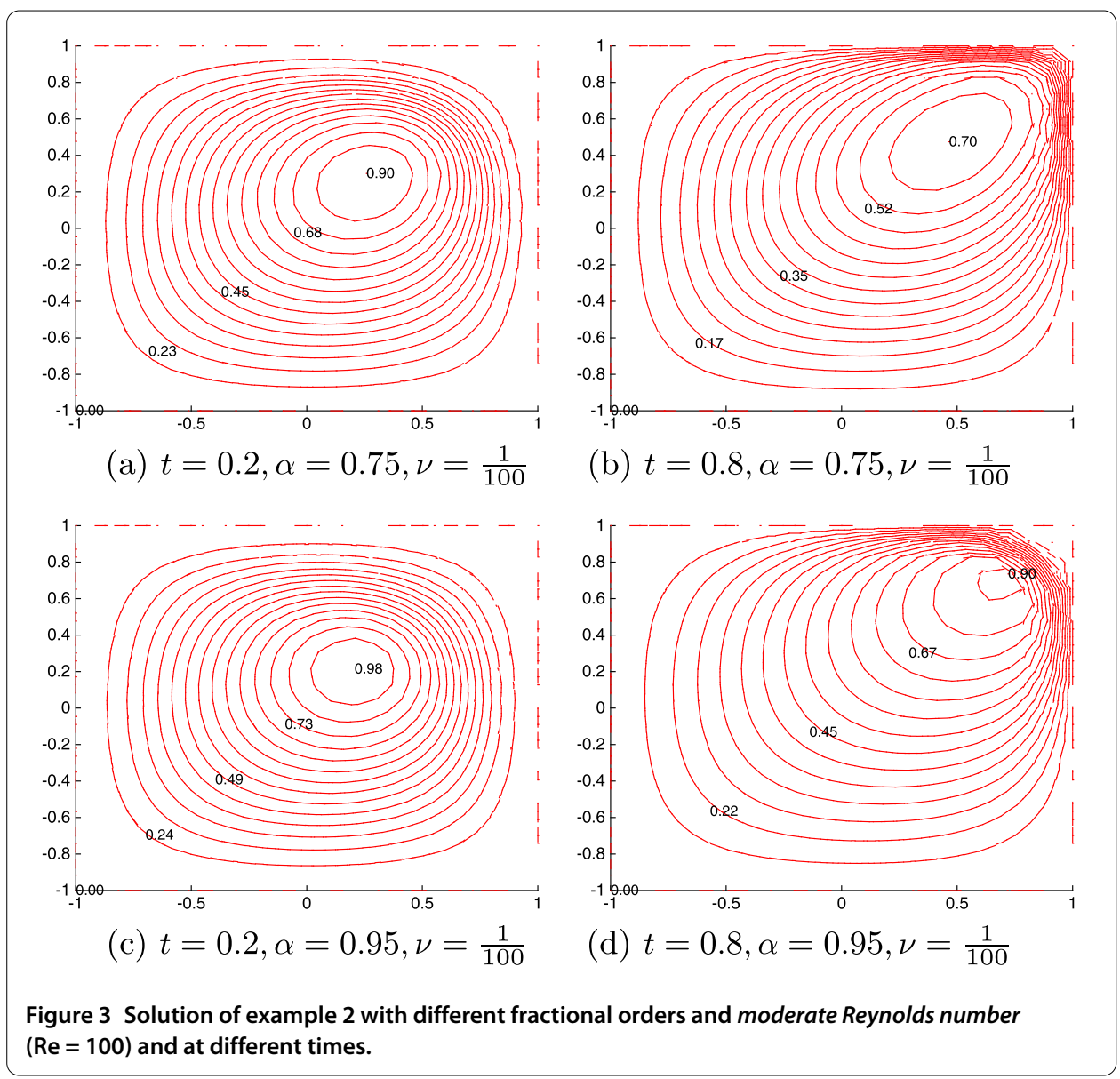

fractional Fick law: a large order of fractional derivative in fractional diffusion processes implies slow diffusion [43-45]. Especially from Figures 1 and 3, we see that the peak of the solution with $\alpha=0.75$ moves to the boundary faster than that with $\alpha=0.95$.

Remark 1 We have mentioned that the fractional Burgers equation does have more interesting dynamical properties and modeling advantages than the conventional Burgers equation [27-29, 32, 34]. Comparing with integer-order Burgers models, fractional ones (e.g., with high Reynolds numbers) present rapid degeneracy; see the differences of dynamics between Figures 1-3(a)(b) and Figures 1-3(c)(d). We could also clearly see that the smaller the order of fractional derivative, the faster the diffusion process of Burgers' equation proceeds. Therefore, the fractional Burgers equation can have more applicable possibilities in traffic flow, conservation law modeling, and turbulence theory. This shall be the fundamental difference between classic and fractional Burgers equations, as well as the basic motivation to further study two-dimensional and three-dimensional temporal and spatial fractional Burgers equations.

\section{Conclusion remarks}

In this paper, we propose a time-fractional two-dimensional Burgers model which contains a Caputo fractional derivative with order $\alpha \in(0,1]$. When $\alpha=1$, the proposed model reduces to the classic Burgers model. We analyze the conservation and dissipation prop- 
erty for both viscid and inviscid fractional Burgers models. To catch the shock wave phenomenon at the wavefront of the fractional Burgers model with high Reynolds number, we implement a mixed algorithm involving time-stepping on temporal variables and the DGM on spatial variables. Numerical simulations are given, which show that the fractional Burgers model preserves the basic property that a shock wave type solution can be found. Meanwhile it exhibits more interesting and rich dynamical behavior by varying the order of fractional derivatives and the Reynolds number. We believe that the shape of the spatial domain may change the dynamics of the fractional Burgers equation, since the shape and volume of the domain determine the differences of eigenvalues of the fractional Burgers equation. More details as regards the fractional Burgers equations (time- and/or space-fractional cases) shall be further studied in the near future.

\section{Acknowledgements}

This work was partially supported by the National Science Foundation of China (No. 11501581), the Youth Foundation of Jiangsu Province (No. BK20170628), the Fundamental Research Funds for the Central Universities (No. 0203-14380012), the Project (No. 502042032) of Central South University and the China Postdoctoral Science Foundation (No. 2015M570683).

\section{Competing interests}

The authors declare that they have no competing interest.

\section{Authors' contributions}

The authors have made the same contribution. All authors read and approved the final manuscript.

\section{Author details}

${ }^{1}$ School of Mathematics and Statistics, Central South University, Changsha, 410083, P.R. China. ${ }^{2}$ Department of Mathematics, Nanjing University, Nanjing, Jiangsu 210093, P.R. China.

\section{Publisher's Note}

Springer Nature remains neutral with regard to jurisdictional claims in published maps and institutional affiliations.

Received: 18 June 2017 Accepted: 10 October 2017 Published online: 23 October 2017

\section{References}

1. Hopf, E: The partial differential equation $u_{t}+u u_{x}=\mu u_{x x}$. Commun. Pure Appl. Math. 3(3), 201-230 (1950)

2. Evans, LC: Partial Differential Equations. Graduate Studies in Mathematics, vol. 19. American Mathematical Society, Providence (2010)

3. Halabisky, L, Sirovich, L: Evolution of finite disturbances in dissipative gas dynamics. Phys. Fluids 3(16), 360-368 (1973)

4. Takači, A: Mathematical and simulation models of traffic flow. Proc. Appl. Math. Mech. 5, 633-634 (2005)

5. Ohwada, T: Cole-Hopf transformation as numerical tool for the Burgers equation. Appl. Comput. Math. 8(1), 107-113 (2009)

6. Zhang, D, Wei, W, Kouri, DJ, Hoffman, DK: Burgers' equation with high Reynolds number. Phys. Fluids 9(6), 1853-1855 (1997)

7. Wazwaz, A: Multiple-front solutions for the Burgers equation and the coupled Burgers equations. Appl. Math. Comput. 190(2), 1198-1206 (2007)

8. Salas, AHS, Gomez, CAS, Hernańdez, JEC: New abundant solutions for the Burgers equation. Comput. Math. Appl. 58(3), 514-520 (2009)

9. Pandey, KB, Verma, L: A note on Crank-Nicolson scheme for Burgers' equation. Appl. Math. 2(7), 883-889 (2011)

10. Kilbas, AA, Srivastava, HM, Trujillo, JJ: Theory and Applications of Fractional Differential Equations. Elsevier, Amsterdam (2006)

11. Hilfer, R: Applications of Fractional Calculus in Physics. World Scientific, Singapore (2000)

12. Metzler, R, Klafter, J: The random walk's guide to anomalous diffusion: a fractional dynamics approach. Phys. Rep. 33, 1-77 (2000)

13. Sabatier, J, Agrawal, OP, Machado, JA: Advances in Fractional Calculus. Springer, Dordrecht (2007)

14. Agila, A, Baleanu, D, Eid, R, Irfanoglu, B: Applications of the extended fractional Euler-Lagrange equations model to freely oscillating dynamical systems. Rom. J. Phys. 61, 350-359 (2016)

15. Kumar, D, Singh, J, Baleanu, D: A fractional model of convective radial fins with temperature-dependent thermal conductivity. Rom. Rep. Phys. 69(1), Article ID 103 (2017)

16. Yang, XJ, Gao, F, Srivastava, HM: New rheological models within local fractional derivative. Rom. Rep. Phys. 69(3), Article ID 113 (2017)

17. Laskin, N: Principles of fractional quantum mechanics. In: Fractional Dynamics, pp. 393-427. World Scientific, Singapore (2011)

18. Atanacković, TM, Pilipović, S, Stanković, B, Zorica, D: Fractional Calculus with Applications in Mechanics: Vibrations and Diffusion Processes. Wiley, New York (2014) 
19. Gorguis, A: A comparison between Cole-Hopf transformation and the decomposition method for solving Burgers' equations. Appl. Math. Comput. 173(1), 126-136 (2006)

20. Sugimoto, $\mathrm{N}$ : Burgers equation with a fractional derivative: hereditary effects on nonlinear acoustic waves. J. Fluid Mech. 225(4), 631-653 (1991)

21. Zhang, Y, Baleanu, D, Yang, XJ: New solutions of the transport equations in porous media within local fractional derivative. Proc. Rom. Acad., Ser. A: Math. Phys. Tech. Sci. Inf. Sci. 17, 230-236 (2016)

22. Wang, Q: Numerical solutions for fractional KdV-Burgers equation by Adomian decomposition method. Appl. Math. Comput. 182(2), 1048-1055 (2006)

23. Chen, $\mathrm{Y}, \mathrm{An}, \mathrm{HL}$ : Numerical solutions of coupled Burgers equations with time- and space-fractional derivatives. Appl. Math. Comput. 200(1), 87-95 (2008)

24. Guesmia, A, Daili, N: Numerical approximation of fractional Burgers equation. Commun. Math. Appl. 1(2), 77-90 (2010)

25. Bhrawy, AH: A new spectral algorithm for time-space fractional partial differential equations with subdiffusion and superdiffusion. Proc. Rom. Acad., Ser. A: Math. Phys. Tech. Sci. Inf. Sci. 17, 39-47 (2016)

26. Abd-Elhameed, WM, Youssri, YH: Spectral solutions for fractional differential equations via a novel Lucas operational matrix of fractional derivatives. Rom. J. Phys. 61, 795-813 (2016)

27. Çenesiz, Y, Baleanu, D, Kurt, A, Tasbozan, O: New exact solutions of Burgers' type equations with conformable derivative. Waves Random Complex Media 27(1), 103-116 (2017)

28. Johnston, SJ, Jafari, H, Moshokoa, S, Baleanu, D: Laplace homotopy perturbation method for Burgers equation with space-and time-fractional order. Open Phys. 14(1), 247-252 (2016)

29. Bhrawy, AH, Zaky, MA, Baleanu, D: New numerical approximations for space-time fractional Burgers' equations via a Legendre spectral-collocation method. Rom. Rep. Phys. 67(2), 340-349 (2015)

30. Abdelkawy, MA, Zaky, MA, Bhrawy, AH, Baleanu, D: Numerical simulation of time variable fractional order mobile-immobile advection-dispersion model. Rom. Rep. Phys. 67, 773-791 (2015)

31. Nouri, K, Elahi-Mehr, S, Torkzadeh, L: Investigation of the behavior of the fractional Bagley-Torvik and Basset equations via numerical inverse Laplace transform. Rom. Rep. Phys. 68, 503-514 (2016)

32. Li, D, Zhang, C, Ran, M: A linear finite difference scheme for generalized time fractional Burgers equation. Appl. Math. Model. 40(11-12), 6069-6081 (2016)

33. Abdelwahed, HG, El-Shewy, EK, Mahmoud, AA: On the time fractional modulation for electron acoustic shock waves. Chin. Phys. Lett. 34(03), Article ID 035202 (2017)

34. Zhao, ZG, Yu, XJ, Wu, D: Numerical solution of the Burgers' equation by local discontinuous Galerkin method. Appl. Math. Comput. 216(12), 3671-3679 (2010)

35. McLean, W, Mustapha, K: Convergence analysis of a discontinuous Galerkin method for a sub-diffusion equation. Numer. Algorithms 5, 69-88 (2009)

36. Mustapha, K, McLean, W: Piecewise-linear, discontinuous Galerkin method for a fractional diffusion equation. Numer. Algorithms 56, 159-184 (2011)

37. Deng, WH, Hesthaven, JS: Local discontinuous Galerkin methods for fractional diffusion equations. ESAIM: Math. Model. Numer. Anal. 47(6), 1845-1864 (2013)

38. Wei, L, Zhang, X, He, Y: Analysis of a local discontinuous Galerkin method for time-fractional advection-diffusion equations. Int. J. Numer. Methods Heat Fluid Flow 23(4), 634-648 (2013)

39. $\mathrm{Xu}, \mathrm{Q}$, Hesthaven, JS: Discontinuous Galerkin method for fractional convection-diffusion equations. SIAM J. Numer. Anal. 52(1), 405-423 (2014)

40. Xu, Q, Zheng, ZS: Discontinuous Galerkin method for time fractional diffusion equation. J. Inf. Comput. Sci. 10(11) 3253-3264 (2013)

41. Shu, CW: Discontinuous Galerkin methods: general approach and stability. In: Numerical Solutions of Partial Differential Equations Adv. Courses Math. CRM, Barcelona, pp. 149-201. Birkhäuser, Basel (2009)

42. Gorenflo, R, Mainardi, F, Moretti, D, Paradisi, P: Time fractional diffusion: a discrete random walk approach. Nonlinear Dyn. 29, 129-143 (2002)

43. Paradisi, P, Cesari, R, Mainardi, F, Tampieri, F: The fractional Fick's law for non-local transport processes. Physica A 293 130-142 (2001)

44. Neel, MC, Abdennadher, A, Joelson, M: Fractional Fick's law: the direct way. J. Phys. A, Math. Theor. 40, 8299-8314 (2007)

45. Valdes-Parada, FJ, Ochoa-Tapia, JA, Alvarez-Ramirez, J: Effective medium equations for fractional Fick's law in porous media. Physica A 373, 339-353 (2007)

\section{Submit your manuscript to a SpringerOpen ${ }^{\circ}$ journal and benefit from:}

- Convenient online submission

- Rigorous peer review

- Open access: articles freely available online

High visibility within the field

- Retaining the copyright to your article

Submit your next manuscript at $\gg$ springeropen.com 